\section{Kidney \\ Blood Pressure Research}

\title{
Chronic Kidney Disease and Nonalcoholic Fatty Liver Disease Proven by Transient Elastography
}

\author{
Ivana Mikolasevic ${ }^{a}$ Sanjin Rackia Ivan Bubic Ita Jelic $^{a} \quad$ Davor Stimac \\ Lidija Orlic ${ }^{a}$
}

aDepartment of Nephrology and Dialysis, Division of Internal medicine, University Hospital Center Rijeka, Rijeka, Croatia; ${ }^{b}$ Department of Gastroenterology, Division of Internal Medicine, University Hospital Rijeka, Rijeka, Croatia

\section{Key Words}

Chronic kidney disease $・$ Non-alcoholic fatty liver disease $\bullet$ Fibroscan ${ }^{\circledR}$

\begin{abstract}
Background/Aim: Preliminary data suggest an association between chronic kidney disease (CKD) and non-alcoholic fatty liver disease (NAFLD). The aim of this study was to further investigate the association between NAFLD and decreased kidney function. Methods: A total of 62 patients with CKD were enrolled in the study. Liver stiffness was used to detect liver fibrosis and CAP (controlled attenuation parameter) was used to detect and quantify liver steatosis (Fibroscan ${ }^{\circledR}$ ). NAFLD was defined by CAP values $\geq 238 \mathrm{~dB} \cdot \mathrm{m}^{-1}$. Results: CKD stage III was present in 29 patients (46.8\%) and CKD stage IV in 33 patients (53.2\%). Out of 62 CKD patients $53(85.5 \%)$ had NAFLD and of these $14 / 53$ patients (26.4\%) had also liver stiffness $>7 \mathrm{kPa}$. The severity of liver steatosis was positively correlated with serum creatinine $(r=0.399 ; p<0.01)$ and CRP $(r=0.261 ; p<0.05)$ and negatively correlated with eGFR $(r=-0.413 ; p<0.01)$ and serum iron concentration $(r=-0.365 ; p<0.01)$. Conclusion: The results suggest a high prevalence of NAFLD in CKD patients. The severity of liver steatosis is negatively correlated with kidney function. The study documents the value of ultrasonographic elastography as an effective non-invasive screening method for the diagnosis of NAFLD.
\end{abstract}

Copyright (C) 2013 S. Karger AG, Basel

\section{Introduction}

Nonalcoholic fatty liver disease (NAFLD) is one of the most common chronic liver diseases in Western countries. It encompasses a spectrum of conditions with lipid deposition in hepatocytes, ranging from simple steatosis to non-alcoholic steatohepatitis (NASH) i.e. fatty liver with inflammation and hepatocelullar injury with or without fibrosis, advanced 


\section{Kidney \\ Blood Pressure Research}

Kidney Blood Press Res 2013;37:305-310

\begin{tabular}{l|l}
\hline DOI: $10.1159 / 000350158$ & (C) 2013 S. Karger AG, Basel
\end{tabular}

Publisnea onine: september 12, 2013

www.karger.com/kbr

Mikolasevic/Racki/Bubic/Jelic/Stimac/Orlic: Chronic Kidney Disease and Nonalcoholic Fatty Liver Disease

fibrosis and cirrhosis. Today, it is believed that NAFLD represents a hepatic manifestation of the metabolic syndrome (MS). MS and all its clinical traits are highly prevalent in patients with NAFLD. In the recent past the importance of NAFLD and its link to the metabolic syndrome has generated great interest in view of its potential role in the development of atherosclerosis [1-5].

Preliminary data suggest an association between chronic kidney disease (CKD) and NAFLD : the prevalence of CKD was significantly higher in patients with NASH compared to patients without NASH [6, 7]. Furthermore, a recent study documented moderately decreased eGFR and high frequency of microalbuminuria in patients with biopsy proven NASH; the severity of liver damage was correlated with lower eGFR [8]. In the absence of a noninvasive method the diagnosis of NAFLD/NASH was based on the gold standard of liver biopsy orless reliably - on serum liver enzymes or ultrasound imaging.. But even the gold standard of liver biopsy has its limitations: apart from being an invasive procedure, connected with serious complications, it samples only $1 / 50000$ th of the liver and is thus prone to significant sampling error. Today it is still under discussion whether liver biopsy is required to confirm a diagnosis of NAFLD $[1-5,9]$

In view of this dilemma many non-invasive procedures have been intensively applied to detect hepatic steatosis and fibrosis. Recently a novel parameter has been developed, i.e. the Controlled Attenuation Parameter (CAP); it can be quantitated using transient elastography (TE) (Fibroscan $囚$ ) which permits to efficiently separate different grades of severity of steatosis. CAP is based on the properties of ultrasonic signals acquired by the Fibroscan ${ }^{\circledR}$. This diagnostic tool allows to simultaneously measure liver stiffness and CAP in the same liver volume. The volume used for the measurement by the Fibroscan ${ }^{\circledR}$ is 200 times larger than that of a liver biopsy specimen. Therefore the Fibroscan ${ }^{\circledR}$ is used more and more in clinical practice [10].

The relationship between NAFLD and CKD is still poorly understood. The aim of the present study was to evaluate how frequently NAFLD is present in patients with chronic kidney disease. In the present cross-sectional study we assessed the frequency of NAFLD in patients in different stages of CKD. To this end we used transient elastography (Fibroscan ${ }^{\circledR}$ CAP).

\section{Patients and Methods}

\section{Patients}

Sixty-two CKD patients were recruited from the Department of Nephrology and Dialysis. CKD was defined as eGFR(MDRD formula) $\leq 60 \mathrm{ml} / \mathrm{min} / 1.73 \mathrm{~m}^{2}$ for $>3$ months. CKD stages were defined according to the National Kidney Foundation. None of the involved patients had serological evidence of chronic hepatitis B and/or C virus infection, had a history of alcohol abuse of more than $20 \mathrm{~g}$ alcohol per day, had a history of drug treatment causing hepatic steatosis (e.g. corticosteroids, high-dose estrogen, methotrexate or amiodaron within 6 months of enrollment) or had a history of gastrointestinal bypass surgery. Other types of metabolic or autoimmune liver disease were ruled out as well.

\section{Methods}

The body mass index (BMI) was calculated as weight (kg) divided by height (m) squared. Obesity was defined as a BMI of $>25 \mathrm{~kg} / \mathrm{m}^{2}$, diabetes mellitus by fasting glucose $\geq 5.6 \mathrm{mmol} / \mathrm{L}$ or drug treatment, dyslipidemia by triglycerides $\geq 1.7 \mathrm{mmol} / \mathrm{L}$, by drug treatment or by $\mathrm{HDL}<1 \mathrm{mmol} / \mathrm{L}$ in men and $<1.29$ in women. Laboratory data included : blood cell count, serum aspartate aminotransferase (AST), alanine aminotransferase (ALT), $\gamma$-glutamyl transferase (GGT), albumin, fasting glucose, total cholesterol, HDL cholesterol, LDL cholesterol, triglycerides, urea, creatinine, and C-reactive protein (CRP) by standard clinical chemistry techniques. The clinical and laboratory data were collected at the time of transient elastography.

Liver stiffness was selected as the parameter to quantitate liver fibrosis. Furthermore the CAP (controlled attenuation parameter) was used to detect and quantify liver steatosis with the help of Fibroscan ${ }^{\circledR}$ (Echosense, Paris, France). The examination was performed using an M probe (at $3 \mathrm{MHz}$ ) on 


\section{Kidney Blood Pressure Research}

Kidney Blood Press Res 2013;37:305-310

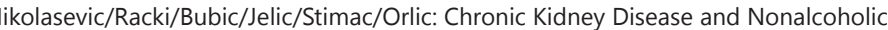
Fatty Liver Disease

Table 1. Demographic characteristics of analyzed pati-

\begin{tabular}{llllc}
\hline \multicolumn{1}{c}{ Characteristic } & Total $(\mathrm{n}=62)$ & CKD III $(\mathrm{n}=29)$ & CKD IV $(\mathrm{n}=33)$ & $\mathrm{p}$ \\
\hline $\begin{array}{l}\text { Age }(\mathrm{y}) \\
\text { Sex }\end{array}$ & $64.9 \pm 8.6$ & $63.3 \pm 9.3$ & $66.4 \pm 7.7$ & $\mathrm{NS}$ \\
$\quad$ Male & $41(66.1 \%)$ & $24(82.8 \%)$ & $17(51.5 \%)$ & $\mathrm{NS}$ \\
Female & $21(33.9 \%)$ & $5(17.2 \%)$ & $16(48.5 \%)$ & 0.04 \\
Etiology: & & & \\
$\quad$ Nondiabetic nephrop. & $37(59.6 \%)$ & $20(69 \%)$ & $13(39.3 \%)$ & \\
$\quad$ Diabetic nephropathy & $25(40.3 \%)$ & $12(41.45)$ & $13(39.4 \%)$ & $\mathrm{NS}$ \\
NAFLD & $53(85.5 \%)$ & $24(45.3 \%)$ & $29(54.7 \%)$ & $\mathrm{NS}$ \\
BMI (kg/m $\left.{ }^{2}\right)$ & $27.19 \pm 2.6$ & $27.8 \pm 2$ & $26.7 \pm 2.7$ & $\mathrm{NS}$ \\
$\begin{array}{l}\text { Hypertension } \\
\text { Diabetes }\end{array}$ & $62(100 \%)$ & $29(100 \%)$ & $33(100 \%)$ & 0.04 \\
Dyslipidemia & $35(56.5 \%)$ & $13(44.8 \%)$ & $22(66.7 \%)$ & $\mathrm{NS}$ \\
\hline *years (y); non-alcoholic fatty liver disease (NAFLD); body mass index (BMI); chronic \\
kidney disease (CKD); non-significant (NS) & & \\
\hline
\end{tabular}

the right lobe of the liver through intercostals spaces with the patients in dorsal decubitus with the right arm in maximal abduction. Ten successful measurements were performed in each patient; only cases with 10 successful acquisitions were taken into account for this study. All scans were performed by the same investigator.

The cut-off value for the diagnosis of liver steatosis was a CAP value $\geq 238 \mathrm{~dB}$. $\mathrm{m}^{-1}$. The cut-off value for the diagnosis of liver fibrosis was liver stiffness $>7 \mathrm{kPa}$.

According to this evaluation, NAFLD was defined by the presence of steatosis with CAP values $\geq 238$ $\mathrm{dB} . \mathrm{m}^{-1}$ regardless of presence or absence of any stage of fibrosis. CAP values between 238 to $258 \mathrm{~dB} . \mathrm{m}^{-1}$ were categorized as steatosis grade 1 (S1), values between 259 to $292 \mathrm{~dB} \cdot \mathrm{m}^{-1}$ as S2, and CAP values $>292 \mathrm{~dB}^{-1} \mathrm{~m}^{-1}$ as steatosis grade 3 (S3) [10].

\section{Statistical Analysis}

Data was performed using descriptive statistics (mean and standard deviation). Categorical variables were tested by chi-square test. Testing the difference of two independent groups was performed using t-test. The Pearson or Spearman correlation coefficient was used to express correlations between variables. P-value $<0.05$ was considered to be statistically significant. Statistical analysis was made using MedCalc statistical software package, version 10 (MedCalc, Mariakerke, Belgium).

\section{Results}

The total cohort of 62 CKD patients comprised 41 men and 21 women with an average age of $64.9 \pm 8.6$ years. 29 patients (46.8\%) had CKD III and 33 patients (53.2\%) CKD IV ; patients with CKD V were not available. Table 1 shows the demographic characteristics of the 62 patients.

Of the 62 CKD patients $53(85.5 \%)$ had CAP > $238 \mathrm{~dB} \cdot \mathrm{m}^{-1}$ and had thus by definition NAFLD.

According to the literature the grades of liver steatosis were defined by CAP values: 10 (18.9\%) patients had grade 1, $14(26.4 \%)$ had grade 2, 29 (54.7\%) had grade 3. Among the patients with NAFLD 14 patients $(26.4 \%)$ had in addition liver stiffness $>7 \mathrm{kPa}$. The number of NAFLD positive patients was not significantly different between CKD stage III and CKD stage IV. The severity of liver steatosis was positively correlated with the serum creatinine concentration, the systolic blood pressure and the CRP concentration; it was negatively correlated with eGFR and serum iron levels (Table 2. and Figure 1.).

The presence of liver fibrosis (defined by liver stiffness $>7 \mathrm{kPa}$ ) in the patients with CAP $\geq 238 \mathrm{dBm}^{-1}$ was not significantly correlated with the eGFR. 


\section{Kidney \\ Blood Pressure Research}

Table 2. Correlation of CAP values (liver steatosis) with laboratory and clinical data
Kidney Blood Press Res 2013;37:305-310

\begin{tabular}{l|l}
\hline DOI: 10.1159/000350158 & (c 2013 S. Karger AG, Basel \\
\hline
\end{tabular}

Publisned online: September 12, 2013

www.karger.com/kbr

Mikolasevic/Racki/Bubic/Jelic/Stimac/Orlic: Chronic Kidney Disease and Nonalcoholic Fatty Liver Disease

\begin{tabular}{|c|c|c|c|c|}
\hline & Mean \pm SD & $\mathrm{r}$ & & $\mathrm{p}$ \\
\hline Hemoglobin (g/L) & $123.3 \pm 16.6$ & 0,001 & NS & \\
\hline Iron $(\mu \mathrm{mol} / \mathrm{L})$ & $13.5 \pm 3.3$ & -0.365 & & $<0.01$ \\
\hline RDW (\%) & $14.8 \pm 1.3$ & 0.120 & NS & \\
\hline Urea (mmol/L) & $14.7 \pm 6.4$ & 0.121 & NS & \\
\hline Creatinine $(\mu \mathrm{mol} / \mathrm{L})$ & $198.7 \pm 67.5$ & 0.399 & & $<0.01$ \\
\hline eGFR $\left(\mathrm{ml} / \mathrm{min}\right.$ per $\left.1,73 \mathrm{~m}^{2}\right)$ & $32.8 \pm 13.6$ & -0.413 & & $<0.01$ \\
\hline Total cholesterol (mmol/L) & $4.5 \pm 1.1$ & 0.239 & NS & \\
\hline Triglycerides (mmol/L) & $1.9 \pm 0.8$ & 0.229 & NS & \\
\hline AST (IU/L) & $20.7 \pm 8.5$ & 0.022 & NS & \\
\hline ALT (IU/L) & $21.3 \pm 10$ & 0.016 & NS & \\
\hline ALP (IU/L) & $90.8 \pm 30.1$ & 0.111 & NS & \\
\hline$\gamma \mathrm{GT}(\mathrm{IU} / \mathrm{L})$ & $31.8 \pm 36.2$ & 0.189 & NS & \\
\hline CRP (mg/L) & $4.2 \pm 5.1$ & 0.261 & & $<0.05$ \\
\hline Age (y) & $64.9 \pm 8.6$ & -0.108 & NS & \\
\hline BMI (kg/m2) & $27.2 \pm 2.6$ & 0.133 & NS & \\
\hline
\end{tabular}

*red blood cell distribution (RDW); estimated glomerular filtration rate (eGFR); aspartate aminotransferase (AST); alanin aminotransferase (ALT); $\gamma$-glutamyltransferase (GGT); C-reactive protein (CRP); systolic blood pressure (SP); diastolic blood pressure (DP); years (y); body mass index (BMI)

\section{Disscusion}

To our knowledge, this is the first study using transient elastography as a noninvasive approach for the detection of NAFLD in CKD patients. Our results demonstrate that CKD patients have a high prevalence of NAFLD $(85.5 \%)$ defined by transient elastography. In our study a significant number of patients had the metabolic syndrome. These results in agreement with past observations that diabetes mellitus type 2 , dyslipidemia and obesity all contribute to the development of NAFLD. It is believed that approximately $90 \%$ of patients with NAFLD have more than one component of the metabolic syndrome: in our study $35-75 \%$ of patients met all diagnostic criteria of the metabolic syndrome [1-5]. We found that the severity of liver steatosis, defined by CAP values, was positively correlated with serum creatinine levels and negatively correlated with eGFR. The frequency of NAFLD between CKD stages III and IV was not significantly different, probably a result of the relatively small number of patients and because NAFLD positive patients were included regardless of the severity of liver steatosis. These findings are in accordance with recent work by Targher et al. [6] which showed that patients with biopsy

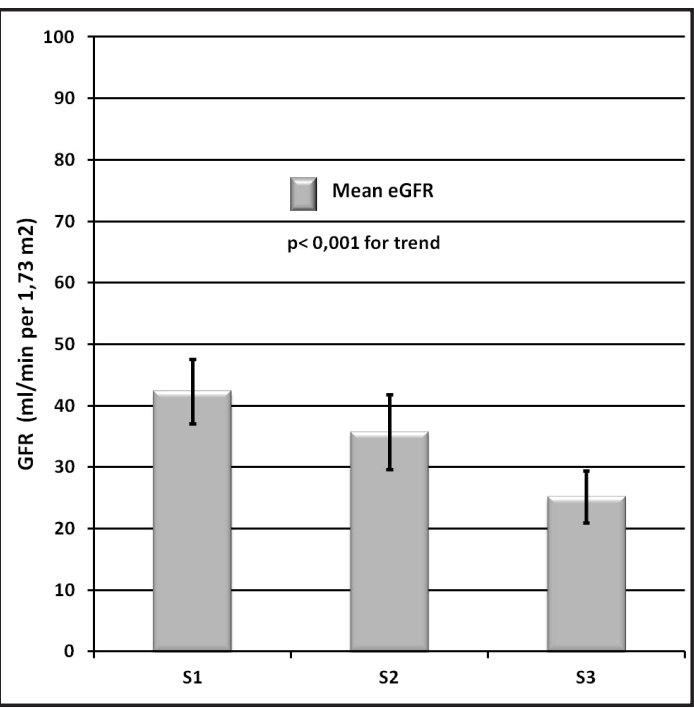

Fig. 1. Adjusted means $( \pm S D)$ of eGFR in relation to the severity of liver steatosis (NAFLD). Steatosis grade increasing from S1 to S3 in CKD patients with Fibroscan $\AA$-CAP proven NAFLD. P value for the trend is assessed by analysis of covariance. 


\section{Kidney Blood Pressure Research}

Kidney Blood Press Res 2013;37:305-310

\begin{tabular}{l|l}
\hline DOI: $10.1159 / 000350158$ & (C) 2013 S. Karger AG, Basel
\end{tabular}

Publisned onIIne: September 12, 2013

www.karger.com/kbr

Molasevic/Racki/Bubic/Jelic/Stimac/Orlic: Chronic Kidney Disease and Nonalcoholic Fatty Liver Disease

proven NASH have a moderately decreased eGFR and that the severity of NASH histology is associated with decreased kidney function. Our results are also corroborated by recent observational studies which showed that the presence of NAFLD is strongly associated with an increased prevalence of CKD [7, 11-12]. Furthermore, Yilmaz et al. [13] showed a positive correlation between microalbuminuria and liver fibrosis in nondiabetic patients with NAFLD. In our study, we didn't find any association between eGFR levels and the severity of liver stiffness. In all of these studies NAFLD was detected by either liver enzymes, liver ultrasound or by a liver biopsy. But it is important to note that the aminotransferase levels that are used as a marker of liver damage are normal in approximately half of all patients with NAFLD; therefore normal values do not exclude NAFLD and liver fibrosis. In our study none of the analyzed liver tests showed a significant correlation with the presence of NAFLD. The sensitivity of the ultrasonography for detection of hepatic steatosis is between $93 \%$ and $100 \%$ if the fat content in hepatic parenchyma is $>33 \%$. But it is a relatively subjective method and is therefore prone to sampling error. Liver biopsy remains the gold standard in the diagnosis of NAFLD, but it is an invasive procedure and there is still no consensus whether the diagnosis of NAFLD should routinely be confirmed by liver biopsy [1-5].

It is important to identify factors that increase the risk of progression of CKD. Today, there is growing evidence that suggests that the metabolic syndrome (MS) is an important and potentially pathogenetically relevant factor for the genesis and progression of chronic kidney disease. In our study a majority of patients had MS and presumably this is the reason why so many patients had NAFLD. It has been well documented in the literature that there is a positive relationship between insulin resistance and CKD $[14,15]$. Insulin resistance is a key pathophysiological mechanism in the metabolic syndrome ; it presumably it also plays a crucial role in the pathogenesis of NAFLD [16]. As mentioned, NAFLD is closely related to the metabolic syndrome and its manifestations, i.e. obesity, diabetes mellitus type 2, dyslipidemia and hypertension. NAFLD represents a liver manifestation of the metabolic syndrome and is an independent predictor of cardiovascular disease. It is believed that subchronic liver inflammation in NAFLD/NASH contributes to enhanced oxidative stress; numerous studies documented a strong link between the severity of NAFLD and the degree of oxidative stress [1-5]. Those studies showed an increase in serum oxidative markers and a decrease in antioxidant molecules in patients with NAFLD. The levels of these markers correlate with the severity of liver disease and insulin resistance [16]. In recent studies serum iron levels were negatively correlated with CAP values pointing to a potential role of chronic inflammation. In the present study, the inflammation marker CRP was positively correlated with CAP values. This correlation might be relevant to explain the inverse correlation between eGFR and liver steatosis. One cannot exclude the possibility that an inflammatory state in NAFLD aggravates CKD progression.

For now, we can only speculate about the implications of these observations and further prospective and randomized trials are needed.

The underlying mechanism by which NAFLD might promote chronic kidney disease remains unknown. It may simply reflect the fact that NAFLD and CKD share some important metabolic risk factors (diabetes mellitus, obesity, dyslipidemia and hypertension); on the other hand, NAFLD might further promote insulin resistance, thus aggravating CKD progression. Finally inflammation and oxidative stress associated with NAFLD may further promote progression, thus establishing a fatal link between liver and kidney.

Our study has certain limitations. The cross-sectional format of our study does not allow conclusions whether the link between CKD and NAFLD is causal. Secondly, we do not have a control group of nonsteatotic patients. Thirdly, we did not use liver biopsy to prove the diagnosis of NAFLD and compare the histological features of liver biopsy samples with the findings by Fibroscan $®$-CAP.

In conclusion, our results suggest that NAFLD is highly prevalent in CKD patients. The severity of liver steatosis is negatively correlated with the kidney function. Whether interventions to interfere with the fatal frequent connection of CKD with obesity, hypertension, diabetes and dyslipidemia would reduce the risk of NAFLD is not known. The main finding of 


\section{Kidney \\ Blood Pressure Research}

Kidney Blood Press Res 2013;37:305-310

\begin{tabular}{l|l}
\hline DOI: 10.1159/000350158 & (c) 2013 S. Karger AG, Basel
\end{tabular}

Publisned online: September 12, 2013

www.karger.com/kbr

Mikolasevic/Racki/Bubic/Jelic/Stimac/Orlic: Chronic Kidney Disease and Nonalcoholic Fatty Liver Disease

our study is the finding that ultrasonographic elastography (FibroScan ${ }^{\circledR}-\mathrm{CAP}$ ) provides the opportunity of non-invasive screening for NAFLD in CKD patients.

\section{Acknowledgements}

The authors gratefully acknowledge the careful editing of the manuscript by Prof. Dr. med, Dr. h.c. mult. Eberhard Ritz, Heidelberg.

\section{References}

1 Dowman JK, Tomlinson JW, Newsome PN: Systematic review: the diagnosis and staging of non-alcoholic fatty liver disease and non-alcoholic steatohepatitis. Aliment Pharmacol Ther 2011;33:525-540.

2 Targher G, Arcaro G: Non-alcoholic fatty liver disease and increased risk of cardiovascular disease. Atherosclerosis 2007;191:235-240.

3 Farrel GC, Larter CZ: Nonalcoholic fatty liver disease: from steatosis to cirrhosis. Hepatology 2006;43:S99-S112.

-4 Starley BQ Calcagno CJ, Harrison SA: Nonalcoholic fatty liver disease and hepatocellular carcinoma: a weighty connection. Hepatology 2010;51:1820-1832.

-5 Kim CH, Younossi ZM: Non alcoholic fatty liver disease: a manifestation of metabolic syndrome. Cleve Clin J Med 2008;75:721-728.

-6 Targher G, Bertolini L, Rodella S, Lippi G, Zoppini G, Chonchol M: Relationship between kidney function and liver histology in subjects with nonalcoholic steatohepatitis. Clin J Am Soc Nephrol 2010;5:2166-2171.

7 Hamad AA, Khalil AA, Connolly V, Ahmed MH: Relationship between non-alcoholic fatty liver disease and kidney function: a communication between two organs that needs further exploration. Arab J Gastroenterol 2012;13:161-165.

>8 YYasui K, Sumida Y, Mori Y, Mitsuyoshi H, Minami M, Itoh Y, Kanemasa K, Matsubara H, Okanoue T, Yoshikawa T: Nonalcoholic steatohepatitis and increased risk of chronic kidney disease. Metabolism 2011;60:735-739.

-9 Martinez SM, Crespo G, Navasa M, Forns X: Noninvasive assessment of liver fibrosis. Hepatology 2011;53:325-335.

10 Sasso M, Beaugrand M, de Ledinghen V, Douvin C, Marcellin P, Poupon R, Sandrin L, Miette V: Controlled attenuation parameter (CAP): a novel VCTE guided ultrasonic attenuation measurement for the evaluation of hepatic steatosis: preliminary study and validation in a cohort of patients with chronic liver disease from various causes. Ultrasound Med Biol 2010;36:1825-1835.

11 Targher G, Kendrick J, Smits G, Chonchol M: Relationship between serum gamma-glutamyltransferase and chronic kidney disease in the United States adult population. Findings from the National Health and Nutrition Examination Survey 2001-2006. Nutr Metab Cardiovasc Dis 2010;20:583-590.

-12 Targher G, Bosworth C, Kendrick J, Smits G, Lippi G, Chonchol M: Relationship of serum bilirubin concentrations to kidney function and albuminuria in the United States adult population. Findings from the National Health and Nutrition Examination Survey 2001-2006. Clin Chem Lab Med 2009;47:1055-1062.

-13 Yilmaz Y, Alahdab YO, Yonal O, Kurt R, Kedrah AE, Celikel CA, Ozdogan O, Duman D, Imeryuz N, Avsar E, Kalayci C: Microalbuminuria in nondiabetic patients with nonalcoholic fatty liver disease: Association with liver fibrosis. Metabolism 2010;59:1327-1330 .

14 Chen J, Muntner P, Hamm LL, Fonseca V, Batuman V, Whelton PK, He J: Insulin resistence and risk of chronic kidney disease in nondiabetic US adults. J Am Soc Nephrol 2003;14:469-477.

15 Chen J, Muntner P, Hamm LL, Jones DW, Batuman V, Fonseca V, Whelton PK, He J: The metabolic syndrome and chronic kidney disease in U.S. adults. Ann Intern Med 2004;140:167-174.

16 Milic S, Stimac D: Nonalcoholic fatty liver disease/steatohepatitis: epidemiology, pathogenesis, clinical presentation and treatment. Dig Dis 2012;30:158-162. 